\title{
DITERPENOS CASBANOS E ACETOFENONAS DE Croton nepetaefolius (EUPHORBIACEAE)
}

Hélcio Silva Santos e Francisca Maria Rodrigues Mesquita

Centro de Ciências Exatas e Tecnologia, Universidade Estadual Vale do Acaraú, 62040-370 Sobral - CE, Brasil

Telma Leda G. Lemos* e Francisco Jose Queiroz Monte

Departamento de Química Orgânica e Inorgânica, Universidade Federal do Ceará, 60451-970 Fortaleza - CE, Brasil

Raimundo Braz-Filho

Setor de Química de Produtos Naturais, LCQUI, CCT, Universidade Estadual Norte Fluminense, 28015-620 Campos - RJ, Brasil

Recebido em 4/6/07; aceito em 6/9/07; publicado na web em 26/2/08

\begin{abstract}
CASBANE DITERPENES AND ACETOPHENONES OF Croton nepetaefolius (EUPHORBIACEAE). Croton nepetaefolius is an aromatic plant native to the northeast of Brazil where it is extensively used in folk medicine as a sedative, orexigen and antispasmodic agent. The present work deals with the chromatographic analysis of the ethanolic extract of Croton nepetaefolius stalk. It allowed the isolation and characterization of two diterpenoids named 1,4-dihydroxy-2E,6E,12E-trien-5-one-casbane and 4-hydroxy2E,6E,12E-5-one-casbane, two acetophenones named 2-hydroxy-4,6-dimethoxyacetophenone and 2-hydroxy-3,4,6trimethoxyacetophenone and the steroids 3-O- $\beta$-D-glucopiranosylsitosterol and a mixture of $\beta$-sitosterol and stigmasterol. Structural elucidation was done on the basis of spectral data, mainly high field NMR and EIMS.
\end{abstract}

Keywords: casbane diterpenoid; acetophenones; Croton nepetaefolius.

\section{INTRODUÇÃO}

O uso de plantas com fins terapêuticos é uma tradição milenar presente nas culturas de várias nações constituindo, ainda hoje, um recurso alternativo de grande aceitação, não somente nos centros urbanos, mas sobretudo nas pequenas comunidades rurais. Este comportamento vem chamando a atenção da comunidade científica no sentido de comprovar a eficácia e promover o uso seguro desses recursos naturais. ${ }^{1}$ Vale ressaltar que as plantas são fontes naturais de uma infinidade de substâncias químicas que são biossintetizadas com várias finalidades, entre elas, protegê-las contra predadores ou atrair polinizadores. ${ }^{2}$

Estudos realizados com espécies de Croton relatam a presença de alcalóides, ${ }^{3-11}$ flavonóides, ${ }^{12}$ esteróides e triterpenóides, ${ }^{12,13}$ saponinas ${ }^{14,15}$ e taninos. ${ }^{16}$ Os diterpenos, principais constituintes do gênero, possuem atividades biológicas, funções ecológicas e constituem protótipos na síntese de substâncias bioativas. ${ }^{17-20}$ Dentre os diferentes tipos estruturais, os diterpenos casbanos são encontrados em apenas seis espécies da família Euphorbiaceae e merecem destaque por apresentarem atividades anticancerígena e bactericida. ${ }^{21-24}$

Croton nepetaefolius é uma planta aromática endêmica do nordeste brasileiro conhecida popularmente como "marmeleiro sabiá", "marmeleiro cravo" ou "marmeleiro de cheiro". Esta espécie é largamente utilizada na medicina popular como estomáquico, carminativo e no tratamento de cólicas intestinais, sendo esta última ação cientificamente comprovada. ${ }^{25}$ Estudos fitoquímicos anteriores relatam a presença de terpenóides ${ }^{26}$ nesta espécie. Neste trabalho, foi realizada uma reinvestigação fitoquímica de Croton nepetaefolius, que resultou no isolamento dos diterpenos casbeno 1,4-diidroxi-2E,6E,12E-trien-5-ona-casbeno (1) e 4-hidroxi2E,6E,12E-trien-5-ona-casbeno (2), sendo o segundo inédito na literatura. Em adição também foram isolados as acetofenonas 2-

*e-mail: tlemos@dqoi.ufc.br hidroxi-4,6-dimetoxiacetofenona (3), 2-hidroxi-3,4,6-trimetoxiacetofenona (4) e os esteróides 3-O- $\beta$-D-glico-piranosilsitosterol (5), $\beta$-sitosterol (6) e estigmasterol (7).

\section{PARTE EXPERIMENTAL}

\section{Procedimentos experimentais gerais}

As cromatografias de adsorção em coluna foram feitas em gel de sílica 60 (63-200 $\mu \mathrm{m}, 70-230$ mesh, Vetec). O comprimento e diâmetro das colunas variaram de acordo com a quantidade de amostra a ser submetida à cromatografia. Nas cromatografias em camada delgada (CCD) foram utilizadas cromatofolhas de alumínio TLC 20 x $20 \mathrm{~cm}$ com gel de sílica $60 \mathrm{GF}_{254}$ (Merck). As revelações das substâncias nas cromatoplacas analíticas foram realizadas, através da exposição destas, com solução de vanilina, seguida de aquecimento. O aparelho utilizado para a obtenção dos espectros de absorção na região do infravermelho foi o espectrômetro Perkin-Elmer, modelo 1000-FT. Os pontos de fusão foram obtidos em equipamento de microdeterminação digital da Mettler Toledo. Os espectros de ressonância magnética nuclear uni e bidimensionais foram obtidos em espectrômetro Bruker DRX-300 e DPX-500 $\left({ }^{1} \mathrm{H}\right.$ : 300 e $500 \mathrm{MHz} ;{ }^{13} \mathrm{C}$ : 75 e $125 \mathrm{MHz}$ ), utilizando $\mathrm{CDCl}_{3}$ como solvente e TMS como padrão interno. Os espectros de massas foram registrados em espectrômetro de massas, aparelho Shimadzu QP5050A, operando em $70 \mathrm{eV}$.

\section{Material vegetal}

O material vegetal (caule) de Croton nepetaefolius foi coletado no município de Caucaia-Ceará, em maio de 2004. A identificação botânica foi realizada pelo Prof. E. Nunes, da Universidade Federal do Ceará. A exsicata da espécie encontra-se depositada no Herbário Prisco Bezerra-UFC sob número 33.582. 


\section{Extração e isolamento}

$\mathrm{O}$ caule $(5 \mathrm{~kg})$, seco à temperatura ambiente, foi triturado e submetido à extração a frio por 3 dias com etanol. A solução obtida foi destilada sob pressão reduzida fornecendo o extrato etanólico $(58,2 \mathrm{~g})$, que foi adsorvido em gel de sílica e submetido à coluna cromatográfica, utilizando-se eluentes: hexano (F 1-15), hexano/ acetato de etila (1:1 F 16-25), acetato de etila (F 26-40) e etanol (F 41-48), obtendo-se um total de 48 frações, de $100 \mathrm{~mL}$ cada. As frações hexânicas (F 1-15) 22,5 g foram reagrupadas e recromatografadas com hexano ( $\left.F^{\prime} 1-10\right)$, hexano/acetato de etila (1:1 F' 11-16), acetato de etila (F' 17-21) e etanol (F' 22-25), obtendo-se um total de 25 frações, de $50 \mathrm{~mL}$ cada. As frações (F' 1116) $14,0 \mathrm{~g}$ foram reagrupadas e submetidas a novo tratamento cromatográfico com hexano ( $F$ " 1$)$, hexano/acetato de etila (9:1 F" 2-5; 8:2 F" 6-15; 7:3 F" 16-32) e acetato de etila (F" 33) obtendose um total de 33 frações, de $25 \mathrm{~mL}$ cada. As frações (F” 10-13), obtidas com hexano/acetato de etila (8:2), reunidas forneceram o diterpeno 1,4-diidroxi-2E,6E,12E-trien-5-ona-casbeno (3,0 g). As frações (F" 7-8) 7,0 g foram reanidas e recromatografadas com hexano (F"' 1-2), hexano/acetato de etila (8:2 F"' 3-4), acetato de etila (F"' 5-10) e etanol (F"' 11) obtendo-se um total de 11 frações, de $50 \mathrm{~mL}$ cada. As frações (F"' 5-10) 4,0 g foram reagrupadas e recromatografadas com hexano/acetato de etila (9:1 F'"' 1-2; 8:2 F"', 3-7; 6:4 F"', 8-14; 4:6 F"'" 15-22) e acetato de etila (F", 23) obtendo-se um total de 23 frações, de $25 \mathrm{~mL}$ cada. As frações (F", 11-12), obtidas com hexano/acetato de etila (6:4), reunidas forneceram o diterpeno 4-hidroxi-2E,6E,12E-trien-5-ona-casbeno (70,0 $\mathrm{mg})$. As frações hexano/acetato de etila (1:1 F 16-25) 10,8 g foram reagrupadas e recromatografadas com hexano ( $\left.F^{\prime} 1\right)$, hexano/acetato de etila (9:1 F' 2-10; 7:3 F' 11-13; 1:1 F' 14-15) e acetato de etila (F' 16), obtendo-se um total de 16 frações, de $100 \mathrm{~mL}$ cada. As frações (F' 10-13) e (F' 16) obtidas com hexano/acetato de etila $(7: 3)$ e acetato de etila, respectivamente, reunidas forneceram a acetofenona 2-hidroxi-4,6-dimetoxiacetofenona (4,0 g, p.f 77-78,5 ${ }^{\circ} \mathrm{C}$ ) e o esteróide 3-O- $\beta$-D-glicopiranosilsitosterol $(80,0 \mathrm{mg}$, p.f $285-287^{\circ} \mathrm{C}$ ). As Frações (F' 14-15) 4,8 g foram submetidas a novo tratamento cromatográfico com hexano ( $F$ " 1-5), hexano/acetato de etila (8:2 F" 6-10; 1:1 F" 11-15; 2:8 F" 16-30) e acetato de etila (F" 31-40) obtendo-se um total de 40 frações, de $50 \mathrm{~mL}$ cada. As frações (F" 6-10), obtidas com hexano/acetato de etila (8:2), reunidas forneceram a acetofenona 2-hidroxi-3,4,6-dime-toxiacetofenona (100 mg, p.f $\left.108-110,6^{\circ} \mathrm{C}\right)$. As frações (F” 14-15) $2,5 \mathrm{~g}$ foram reunidas e recromatografadas com hexano ( $\mathrm{F}^{\prime \prime}$ ' 1 ), hexano/acetato de etila (9:1 F"' 2-20; 8:2 F" 21-45; 7:3 F" 4670; 1:1 F"' 71-80; 4:6 F" 81-100; 2:8 F"' 101-110) e acetato de etila (F"' 111-121) obtendo-se um total de 121 frações, de $25 \mathrm{~mL}$ cada. As frações (F"' 81-90), obtidas com hexano/acetato de etila (4:6), reunidas forneceram os esteróides $\beta$-sitosterol e estigmasterol (40,0 mg).

\section{Propriedades físicas e espectroscópicas}

1,4-diidroxi-2E,6E,12E-trien-5-ona-casbeno (1): óleo verde, I.V. $\left(\mathrm{KBr}, v_{\max } \mathrm{cm}^{-1}\right): 3400,2920,1660,1618,1020,756$; EMIE $\mathrm{m} / \mathrm{z}$ (\%) $318\left([\mathrm{M}]^{+}\right.$, ausente), 300 (2), 282 (2), 150 (14), 135 (30), 121 (22), 107 (44). RMN ${ }^{1} \mathrm{H}^{13} \mathrm{C}$, Tabela 1.

4-hidroxi-2E,6E,12E-trien-5-ona-casbeno (2): óleo verde, I.V. (KBr, $\left.v_{\max } \mathrm{cm}^{-1}\right): 3508,2916,1697,1649,1455,1116,753$; EMIE $\mathrm{m} / \mathrm{z}$ (\%) $302\left([\mathrm{M}]^{+}, 2\right), 149(18), 135$ (15), 121 (22), 107 (24). RMN ${ }^{1} \mathrm{H}$ ${ }^{13} \mathrm{C}$, Tabela 1.

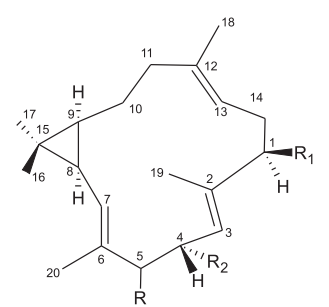<smiles>[2H]c1cc(C(C)=O)c(O)c([O-])c1O</smiles>

(1) $\mathbf{R}==\mathbf{O} ; \mathbf{R}_{1}=\mathbf{R}_{\mathbf{2}}=\mathrm{OH}$

(2) $\quad \mathrm{R}=\mathrm{O}$; $\mathrm{R}_{1}=\mathrm{H} ; \mathrm{R}_{2}=\mathrm{OH}$ (4) $\mathrm{R}=\mathrm{OMe}$

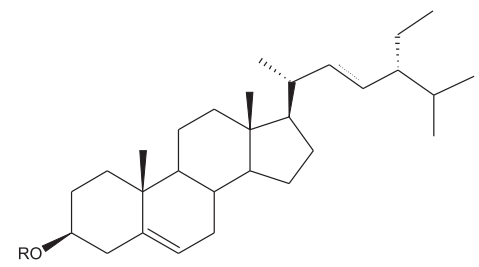

( 5 ) $\mathrm{R}=3-\mathrm{O}-\beta-\mathrm{D}-\mathrm{glicopiranosil}$

(6) $R=H, 22,23$-diidro

(7) $\mathrm{R}=\mathrm{H}, \Delta^{22-23}$

Figura 1. Substâncias isoladas do extrato etanólico do caule de Croton nepetaefolius $1-7$

\section{RESULTADOS E DISCUSSÃO}

Os compostos $\mathbf{1}$ e $\mathbf{2}$ foram isolados a partir da fração hexânica do extrato etanólico de $C$. nepetaefolius como óleos densos esverdeados. Por intermédio dos espectros de RMN ${ }^{13} \mathrm{C}$ (HBBD e DEPT 135) verificou-se a presença de 20 sinais de carbonos para ambos. O composto 1, isolado anteriormente dessa espécie, foi identificado como sendo o diterpeno 1,4-diidroxi-2E,6E,12E-trien-5ona-casbeno cujos dados espectrais de $\mathrm{RMN}{ }^{1} \mathrm{H}, \mathrm{RMN}{ }^{13} \mathrm{C}$ e IV estão em acordo com valores da literatura. ${ }^{26}$ Por outro lado, a comparação dos deslocamentos químicos (Tabela 1) dos átomos de hidrogênio e carbono de $\mathbf{2}$ com os do composto $\mathbf{1}$ indicou a existência de um sistema casbânico similar. Vale destacar nos espectros de RMN ${ }^{13} \mathrm{C}$ (HBBD e DEPT) de 2, os sinais em $\delta_{\mathrm{C}} 142,71$ (C), 125,54 $(\mathrm{CH}), 134,56(\mathrm{C}), 145,06(\mathrm{CH}), 136,50(\mathrm{C}), 124,10(\mathrm{CH})$ indicando três ligações olefínicas trissubstituídas, compatível com os sinais no espectro de $\mathrm{RMN}{ }^{1} \mathrm{H}$ para três hidrogenios olefínicos em $\delta_{\mathrm{H}}$ $5,08(\mathrm{~d}, 9,1), 6,34(\mathrm{~d}, 10,5)$ e 4,83 (d, 8,2), condição semelhante à observada em 1 (Tabela 1). A conectividade dos carbonos metínicos com seus respectivos hidrogênios foi claramente observada no espectro ${ }^{1} \mathrm{H}_{-}{ }^{13} \mathrm{C}$ HMQC. Vale também ressaltar no espectro de RMN ${ }^{13}$ HBBD o sinal compatível com cetona $\alpha, \beta$-insaturada em $\delta_{C} 200,40$ $\left(\delta_{C} 199,74\right.$ em 1) e no experimento HMBC, as correlações heteronuclear de hidrogênio e carbono a longa distância entre os carbonos C-2 $\left(\delta_{\mathrm{C}} 142,71\right)$, C-6 $\left(\delta_{\mathrm{C}} 134,56\right)$ e C-12 $\left(\delta_{\mathrm{C}} 136,50\right)$ e os hidrogênios $3 \mathrm{H}-19\left(\delta_{\mathrm{H}} 1,69\right)$, 3H-20 $\left(\delta_{\mathrm{H}} 1,95\right)$ e $3 \mathrm{H}-18\left(\delta_{\mathrm{H}} 1,58\right)$, respectivamente, indicando que três grupos metílicos se encontravam ligados a carbonos olefínicos. Paralelamente, as correlações do carbono quaternário $\mathrm{C}-15\left(\delta_{\mathrm{C}} 27,44\right)$ com os hidrogênios metílicos $3 \mathrm{H}-16\left(\delta_{\mathrm{H}} 1,02\right)$ e $3 \mathrm{H}-17\left(\delta_{\mathrm{H}} 1,17\right)$ e destes com os carbo$\operatorname{nos} \mathrm{C}-8\left(\delta_{\mathrm{C}} 28,45\right)$ e $\mathrm{C}-9\left(\delta_{\mathrm{C}} 36,01\right)$ revelaram que esses dois grupos metílicos encontravam-se ligados ao carbono quaternário $\mathrm{C}-15$ do anel ciclopropila. Todos esses sinais são característicos de diterpenos com esqueleto casbano com anel macrocíclico de 
Tabela 1. Dados de $\mathrm{RMN}^{1} \mathrm{H}$ e ${ }^{13} \mathrm{C}$ de 1 e $2\left(\mathrm{CDCl}_{3}\right)$, incluindo-se resultados de correlação heteronuclear ${ }^{1} \mathrm{H}_{-}{ }^{13} \mathrm{C}_{-} \mathrm{COSY}-{ }^{1} \mathrm{~J}_{\mathrm{CH}}(\mathrm{HMQC})$ e ${ }^{1} \mathrm{H}-$ ${ }^{13} \mathrm{C}-\mathrm{COSY}{ }^{-} \mathrm{J}_{\mathrm{CH}}(\mathrm{HMBC})$. Deslocamentos químicos em $\delta_{\mathrm{C}}$ e $\delta_{\mathrm{H}}(\mathrm{ppm})$ e constantes de acoplamento ( $J$, entre parênteses) em Hz

\begin{tabular}{|c|c|c|c|c|c|c|}
\hline & & 1 & & & & \\
\hline & & & $\mathrm{HM}$ & & $\mathrm{HMB}$ & \\
\hline & $\delta_{\mathrm{C}}$ & $\delta_{\mathrm{H}}$ & $\delta_{\mathrm{C}}$ & $\delta_{\mathrm{H}}$ & ${ }^{2} \mathbf{J}_{\mathrm{CH}}$ & ${ }^{3} \mathbf{J}_{\mathrm{CH}}$ \\
\hline 1 & 77,64 & $4,09(\mathrm{dd}, 9,6,6,2)$ & 38,91 & $2,20(\mathrm{~m})$ & & \\
\hline 2 & 144,02 & & 142,71 & & $3 \mathrm{H}-19$ & \\
\hline 3 & 125,48 & $5,14(\mathrm{~d}, 9,4)$ & 124,54 & $5,08(\mathrm{~d}, 9,1)$ & & $3 \mathrm{H}-19$ \\
\hline 4 & 67,82 & $5,24(d, 9,4)$ & 68,63 & $5,23(\mathrm{~d}, 9,1)$ & & \\
\hline 5 & 199,74 & & 200,40 & & & \\
\hline 6 & 134,61 & & 134,56 & & $3 \mathrm{H}-20$ & \\
\hline 7 & 145,11 & $6,25(\mathrm{~d}, 10,4)$ & 145,06 & $6,34(d, 10,5)$ & & \\
\hline 8 & 28,08 & $1,50(\mathrm{dd}, 10,4,8,3)$ & 28,45 & $1,55(\mathrm{~m})$ & & $3 \mathrm{H}-16 ; 3 \mathrm{H}-17$ \\
\hline 9 & 35,67 & $1,23-1,15(\mathrm{~m})$ & 36,01 & $1,20(\mathrm{~m})$ & & $3 \mathrm{H}-16 ; 3 \mathrm{H}-17$ \\
\hline 10 & 25,36 & $1,23-1,15(\mathrm{~m}) 0,79-0,72(\mathrm{~m})$ & 26,13 & $0,90(\mathrm{~m})$ & & \\
\hline 11 & 39,85 & $2,27-2,03(\mathrm{~m}) 1,71-1,68(\mathrm{~m})$ & 40,02 & $1,80(\mathrm{~m})$ & & \\
\hline 12 & 137,39 & & 136,50 & & $3 \mathrm{H}-18$ & \\
\hline 13 & 119,72 & $4,66(\mathrm{dd}, 6,3)$ & 124,10 & $4,83(\mathrm{dl}, 8,2)$ & & $3 \mathrm{H}-18$ \\
\hline 14 & 31,66 & $2,27(\mathrm{~m})$ & 24,14 & $2,28-2,00(\mathrm{~m})$ & & \\
\hline 15 & 27,73 & & 27,44 & & $3 \mathrm{H}-16 ; 3 \mathrm{H}-17$ & \\
\hline 16 & 16,12 & 0,99 (s) & 16,16 & $1,02(\mathrm{~s})$ & & $3 \mathrm{H}-17$ \\
\hline 17 & 29,25 & $1,13(\mathrm{~s})$ & 29,38 & $1,17(\mathrm{~s})$ & & $3 \mathrm{H}-16$ \\
\hline 18 & 15,43 & $1,56(\mathrm{~s})$ & 15,69 & $1,58(\mathrm{sl})$ & & \\
\hline 19 & 10,15 & $1,66(\mathrm{~s})$ & 15,78 & $1,69(\mathrm{sl})$ & & \\
\hline 20 & 12,08 & $1,90(\mathrm{~s})$ & 12,16 & $1,95(\mathrm{sl})$ & & \\
\hline
\end{tabular}

quatorze membros. ${ }^{27,28}$ Porém, os espectros de RMN ${ }^{13} \mathrm{C}$ e DEPT 135 mostraram a presença de um grupo metilênico a mais na estrutura de 2 através do sinal em $\delta_{\mathrm{C}} 38,91$. Adicionalmente, os espectros de RMN ${ }^{1} \mathrm{He}$ e ${ }^{13} \mathrm{C}$ de 2 não exibem os sinais em $\delta_{\mathrm{H}} 4,09(\mathrm{H}-1)$ e $\delta_{\mathrm{C}} 77,64(\mathrm{C}-1)$, respectivamente, registrados nos espectros de $\mathbf{1}$. Estas observações mostraram que o carbono metínico carbinólico C-1 em 1 se encontra na forma reduzida em 2, ou seja, como carbono metilênico $\left(\delta_{\mathrm{C}} 38,91\right)$, sendo esta a única diferença entre as estruturas 1 e 2. As atribuições dos deslocamentos químicos dos átomos de hidrogênio e carbono foram efetuadas de modo inequívoco pela utilização das técnicas de $\mathrm{RMN}$ unidimensionais $\left({ }^{1} \mathrm{H}\right.$, ${ }^{13} \mathrm{C}$ e DEPT) e bidimensionais $\left({ }^{1} \mathrm{H}-{ }^{13} \mathrm{C}\right.$ HMQC e $\left.{ }^{1} \mathrm{H}-{ }^{13} \mathrm{C} \mathrm{HMBC}\right)$ (Tabela 1). As configurações relativas dos carbonos quirais $\mathrm{C}-4$, C-8 e C-9 de 2 foram determinadas com base na comparação dos dados espectrais de RMN ( ${ }^{1} \mathrm{H} \mathrm{e}{ }^{13} \mathrm{C}$ ) de $\mathbf{2}$ com dados do composto $\mathbf{1}$ tomado como modelo. Assim, os deslocamentos químicos e os valores das constantes de acoplamento dos hidrogênios H-3, H-4, H-7, H-8 e H-9 de 2 e $\mathbf{1}$ são muito próximos (Tabela 1) e consistentes com a mesma estereoquímica dos carbonos quirais correspondentes (C-4, C-8 e C-9) e das ligações duplas ( $\Delta^{2}$-E e $\Delta^{6}$-E) nesses compostos. A comparação dos deslocamentos químicos dos carbonos C-13-C-12, C-15-C-18 e C-20 de 2 com os respectivos carbonos de 1 revelou, praticamente, os mesmos valores, e amparou esta dedução (Tabela 1). As exceções (C-1, C-2, C-13, C-14 e C-19) são justificadas pelos efeitos $(\alpha, \beta$ e $\gamma)$ do grupo hidroxila (ausente em 2) no carbono C-1 de $\mathbf{1}$. Assim, 2 foi identificado como o diterpeno 4-hidroxi-2E,6E,12E-trien-5-ona-casbeno, um metabólito secundário inédito na literatura.

$\mathrm{O}$ composto 3 tem fórmula molecular $\mathrm{C}_{10} \mathrm{H}_{12} \mathrm{O}_{4}$, determinada através do espectro de massas $(\mathrm{m} / \mathrm{z}, 196)$ e dos espectros de $\mathrm{RMN}{ }^{13} \mathrm{C}$ (BB e DEPT 135). A análise dos espectros de RMN ( $\left.{ }^{1} \mathrm{H} \mathrm{e}{ }^{13} \mathrm{C}\right)$, IV e EM, assim como a comparação com os dados dos espectros de RMN ${ }^{1} \mathrm{H}$ e IV de 3 publicados na literatura ${ }^{29}$ permitiram identificar a estrutura como 2-hidroxi-4,6-dimetoxiacetofenona. O composto 4 com fórmula molecular $\mathrm{C}_{11} \mathrm{H}_{14} \mathrm{O}_{5}(\mathrm{~m} / \mathrm{z}, 226)$ tem 30 u.m.a. a mais que $\mathbf{3}$, sugerindo a presença de um terceiro grupo metoxílico em sua estrutura. Esta dedução é comprovada nos espectros de $\mathrm{RMN}{ }^{1} \mathrm{H}\left(\delta_{\mathrm{H}} 3,96\right.$, $3,91$ e 3,83$)$ e ${ }^{13} \mathrm{C}\left(\delta_{\mathrm{C}} 60,86,56,11\right.$ e 55,74$)$. Finalmente, a análise dos espectros (RMN ${ }^{1} \mathrm{H}$ e ${ }^{13} \mathrm{C}$, IV e EM) e a comparação com dados da literatura ${ }^{30}$ permitiram identificar 4 como 2-hidroxi-3,4,6trimetoxiacetofenona. Os esteróides 3-O- $\beta$-D-glicopiranisilsitosterol (5) $\beta$-sitosterol (6) e estigmasterol (7) foram caracterizadas com base nos dados espectrais de $\mathrm{RMN}{ }^{1} \mathrm{H} \mathrm{e}{ }^{13} \mathrm{C}$, envolvendo principalmente a comparação dos deslocamentos químicos dos átomos de carbono com valores descritos na literatura. ${ }^{31}$

\section{AGRADECIMENTOS}

Às instituições financiadoras CNPq, CAPES, FUNCAP e FAPERJ pelas bolsas e auxílios concedidos, ao CENAUREM-UFC pela obtenção dos espectros de RMN e ao Prof. E. R. Silveira DQOIUFC pela coleta do material botânico.

\section{REFERÊNCIAS}

1. Fennell, C. W.; Lindsey, K. L.; McGraw, L.; Sparg, S. G.; Stafford, G. I.; Elgorashi, E. E.; Grace, O. M.; Staden, J.; J. Ethnopharmacol. 2004, 94, 205.

2. Stepp, J. R.; J. Ethnopharmacol. 2004, 92, 163.

3. Sanchez, V.; Sandoval, D.; Herrera, P.; Oquedo, M.; Rev. Cubana Farm. 1982b, 16, 39.

4. Smolenski, S. J.; Silinis, H.; Farnsworth, N. R.; Lloydia 1975, 38, 479.

5. Bandoni, A. L.; Mendiondo, M. E.; Rondina, R. V. D.; Coussio, J. D.; II Econ. Bot. 1976, 30, 161.

6. Sanchez, V.; Sandoval, D.; Rev. Cubana Farm. 1982a, 16, 45.

7. Nakanishi, K.; Sasaki, S. I.; Kiang, A. K.; Goh, J.; Kakisawa, H.; Ohashi, M.; Goto, M.; Watanabe, J. M.; Yokotami, H.; Matsumura, C.; Togashi, M.; Chem. Pharm. Bull. 1965, 13, 882.

8. Gunatilaka, A. A. L.; Sultanbawa, M. U. S.; Balasubramaniam, S.; J. Natl. Sci. Counc. 1980, 8, 187.

9. Collin, M. C. L. A.; Levy, J.; Bull. Soc. Pharma. 1976, 32, 267.

10. Nimmanhemin, S.; Apisariyakul, A.; Bull. Chieng Mai Ass. Med. Sci. 1979, 12,125 . 
11. Arseculeratne, S. N.; Gunatilaka, A. A. L.; Panabokke, R. G.; J. Ethnopharmacol. 1981, 4, 159.

12. Shetty, S. N.; Anika, S. M.; Asuzu, U. I.; Int. J. Crude Drug Res. 1983 , 21,49 .

13. Khier, Y. M.; Salih, A. M.; J. Afr. Med. Pl. 1979, 2, 55.

14. Vera, R.; Smadja, J.; Conan, J. Y.; Plant Med. Phytother. 1990, 24, 50.

15. Sinha, S. K. P.; Dogra, J. V. V.; J. Crude Drug Res. 1985, 23, 77.

16. Cespedes, R.; Chacon, S.; Mora, A. L.; Ing. Cienc. Quim. 1992, 14, 6.

17. Hanson, J. R.; Nat. Prod. Rep. 1995,12, 207.

18. Hanson, J. R.; Nat. Prod. Rep. 1996, 13, 59.

19. Hanson, J. R.; Nat. Prod. Rep. 1997, 14, 245.

20. Hanson, J. R.; Nat. Prod. Rep. 1998, 15, 93.

21. Choi, Y. H.; Kim, J.; Pezzuto, J. M.; Kinghorn, A. D; Farnsworth, N. R.; Tetrahedron Lett. 1986, 27, 5795.

22. Choi, Y. H.; Pezzuto, J. M.; Kinghorn, A. D.; Farnsworth, N. R.; J. Nat. Prod. 1988, 51, 110.
23. Burke, B. A.; Chan, W. R.; Pascoe, K. O.; Blount, J. F.; Manchand, P. S.; J. Chem. Soc., Perkin Trans 1 1981, 10, 2666.

24. Xu, Z. H.; Sun, J.; Xu, R. S.; Qin, G. W.; Phytochemistry 1998, 49, 149.

25. Abdon, A. P. V.; Leal-Cardoso, J. H.; Coelho-de-Souza, A. N.; Morais, S. M.; Santos, C. F.; Braz. J. Med. Biol. Res. 2002, 35, 1215.

26. Vera, L. A.; Monte, F. J. Q.; Braz Filho, R.; J. Nat. Prod. 1990, 53, 1566.

27. Bai, Y.; Yang, Y. P.; Ye, Y.; Tetrahedron Lett. 2006, 47, 6637.

28. Kashman, Y.; Bernart, M. W.; Tischler, M.; Cardellina, J. H.; Boyd, M. R.; J. Nat. Prod. 1994, 57, 426

29. Soares, M. G.; Felippe, A. P. V.; Guimarães, E. F.; Kato, M. J.; Ellena, J.; Doriguetto, A. C.; J. Braz. Chem. Soc. 2006, 17, 1205.

30. Patra, A.; Ghosh, G.; Magn. Reson. Chem. 1987, 25, 734

31. Macari, P. T. A.; Emerenciano, V. P.; Ferreira, Z. M. G. S.; Quim. Nova 1990, 13, 260. 